\title{
Two-Dimensional Fractal Segmentation of Natural Images
}

\author{
Vo Anh ${ }^{1}$, Junji Maeda ${ }^{2}$, Tohru Ishizaka ${ }^{2}$, Yukinori Suzuki ${ }^{2}$, and Quang Tieng ${ }^{1}$ \\ 1 Centre In Statistical Science And Industrial Mathematics, Queensland University \\ of Technology, GPO Box 2434, Brisbane, Q.4001, Australia \\ 2 Department of Computer Science And Systems Engineering, Muroran Institute of \\ Technology, 27-1 Mizumotocho, Muroran 050 Japan
}

\begin{abstract}
This paper presents a method that integrates fractal dimension and edge information into a region growing algorithm for segmentation of natural images. The local fractal dimension is estimated by two methods, one based on the 1-D Fourier-wavelet transform and the other on the 2-D Fourier transform. The algorithm also consists of a technique which stores edge informaiton not on a pixel itself but on a boundary between pixels in the region-edge integrating algorithm in order to use the edge information more effectively and to simplify the algorithm. Experimental results are presented to evaluate the performance of the methods.
\end{abstract}

\section{Introduction}

Image segmentation is an important task in image analysis and computer vision. In particular, it is an essential component in an algorithm for pattern recognition and classification (an example being recognition of road signs for use in a geographic information system). Pentland (1984) showed that natural images are commonly isotropic fractals over a range of scales, and fractional Brownian motion $(\mathrm{fBm})$ can be used to model textured and shaded image regions. The defining characteristic of a fractal surface is its fractal dimension (FD). This characteristic furnishes a scale-invariant description of a natural image and corresponds closely to the intuitive notion of surface roughness.

Because of these desirable properties, the FD is an important feature of a natural image that can be used in an algorithm for its segmentation (see Keller et al. (1989) and Keller and Seo (1990)). However, it is known that the FD alone does not perform a satisfactory segmentation due to its high resolution, hence still yielding a large number of details (see Maeda et al. (1996)). On the other hand, a procedure based on both region and edge information is known to be effective for image segmentation (Pavlidis and Liow (1990)).

Maeda et al. (1996) proposed a method that integrates the FD information and the edge information into a region growing algorithm for natural image segmentation. In this method, the FD is used as a local feature that contains the region information, while the edge information is used to control the region growing algorithm. Maeda et al. (1996) used a Fourier-wavelet method to estimate the FD of a local region of the image. This FD is related to the exponent 
in the power-law form of the spectral density of the random field generating that image (Anh et al. (1996)).

The method of Maeda et al. (1996) in estimating this exponent, and hence the FD, is one-dimensional; that is, each local region is scanned horizontally (or vertically or diagonally) to obtain a time series for the estimation. This 1-D approach is fast to implement, but may not be as efficient as a 2-D approach, which uses information in the entire neighbourhood of each point. This paper proposes a 2-D method to estimate the FD of a local region. The key element is the 2-D isotropic version of $\mathrm{fBm}$, whose spectral density also involves an exponent relating to the FD. Our periodogram-based method to estimate this exponent works well for a local region (even as small as $5 \times 5$ ) because it only requires that portion of the periodogram in a small neighbourhood of frequency 0 . We will compare the performance of this 2-D method with the 1-D Fourier-wavelet method in this paper.

Another component of our segmentation algorithm is based on the technique of edge representation by boundary pixels, called the boundary edge (Maeda et al. (1996)). This technique improves the control ability of the edge information in the region growing algorithm and also simplifies the algorithm.

The local spectral density and the Fourier-wavelet method for the general case of random fields in $\Re^{n}$ are described in Section 2, while Section 3 details the 2-D spectral method of estimating the FD. The segmentation algorithm is outlined in Section 4. The experimental results are reported in Section 5. Finally, some conclusions are drawn in Section 6.

\section{Local spectral density}

In this section, we define the local spectral density and local periodogram of a random field defined on $\Re^{n}$. We will consider the wavelet functions $\psi$ satisfying the usual admissibility condition (Koornwinder (1993), p. 30) and denote

$$
\psi_{a, b}(t)=\frac{1}{a^{\frac{n}{2}}} \psi\left(\frac{t-b}{a}\right), a>0, b, t \in \Re^{n} .
$$

Then the Fourier transform of $\psi_{a, b}(t)$ is

$$
\hat{\psi}_{a, b}(\omega)=a^{\frac{n}{2}} \hat{\psi}(a \omega) e^{-i(b, \omega)}
$$

The Fourier-wavelet transform of a random field $X(t)$ is defined as

$$
\hat{X}_{\psi_{a, b}}(\omega)=\int_{\Re^{n}} X(t) e^{-i(t, \omega)} \overline{\psi_{a, b}(t)} d t, \omega \in \Re^{n}
$$

if it exists. In order to motivate a concept of local spectral density, we assume for the time being that $X(t)$ is stationary with covariance function $R(t)$ and spectral density $f(\omega)$. Then the following spectral representation holds:

$$
E(X(t) X(s))=R(t-s)=\int_{\Re^{n}} e^{i(t-s, \omega)} f(\omega) d \omega .
$$


It now follows that, using (4),

$$
\begin{gathered}
E\left|\hat{X}_{\psi_{a, b}}(\omega)\right|^{2} \\
=\int_{\Re^{n}} \int_{\Re^{n}} E(X(t) X(s)) e^{-i(t-s, \omega)} \psi_{a, b}(t) \overline{\psi_{a, b}(s)} d t d s \\
=\int_{\Re^{n}} \int_{\Re^{n}}\left(\int_{\Re^{n}} e^{i(t-s, \lambda)} f(\lambda) d \lambda\right) e^{-i(t-s, \omega)} \psi_{a, b}(t) \overline{\psi_{a, b}(s)} d t d s \\
=\int_{\Re^{n}} f(\lambda)\left[\left(\int_{\Re^{n}} e^{i(t, \lambda-\omega)} \psi_{a, b}(t) d t\right)\left(\int_{\Re^{n}} e^{-i(s, \lambda-\omega) \overline{\psi_{a, b}(s)}} d s\right)\right] d \lambda \\
=\int_{\Re^{n}} f(\lambda)\left|\hat{\psi}_{a, b}(\lambda-\omega)\right|^{2} d \lambda .
\end{gathered}
$$

In view of (2) and (5), the quantity $\frac{1}{a^{n}} E\left|\hat{X}_{\psi_{a, b}}(\omega)\right|^{2}$ may be considered as the scale-space energy density of $X(t)$. As a result, we define the local spectral density of $X(t)$ as

$$
\lim _{l \rightarrow \infty} \frac{1}{l} \int_{0^{+}}^{l} E\left|\hat{X}_{\psi_{a, b}}(\omega)\right|^{2} \frac{d a}{a^{n+1}}
$$

if the limit exists. Now, by choosing the wavelet function $\psi$ so that

$$
\lim _{l \rightarrow \infty} \frac{1}{l} \int_{0^{+}}^{l}\left|\hat{\psi}_{a, b}(\lambda-\omega)\right|^{2} \frac{d a}{a^{n+1}}=C_{\psi} \delta(\lambda-\omega)
$$

where $C_{\psi}$ is a constant dependent only on $\psi$, and $\delta$ is the Dirac delta function (Koornwinder (1993), p. 30), we obtain from (5) and (7) that

$$
\lim _{l \rightarrow \infty} \frac{1}{l} \int_{0^{+}}^{l} E\left|\hat{X}_{\psi_{a, b}}(\omega)\right|^{2} \frac{d a}{a^{n+1}}=C_{\psi} f(\omega)
$$

using Fubini's theorem. The result (8) suggests a definition of the local periodogram as

$$
I_{\psi}(\omega)=\frac{1}{C_{\psi}} \frac{1}{T^{n} l} \int_{[0, T]^{n}} \int_{0^{+}}^{l}\left|\hat{X}_{\psi_{a, b}}(\omega)\right|^{2} \frac{d a}{a^{n+1}} d b
$$

Remark. For an example of a wavelet function which satisfies condition (7), we may take the Marr wavelet (second derivative of the 1-D Gaussian):

$$
\psi(t)=\left(1-t^{2}\right) e^{-\frac{t^{2}}{2}}, \hat{\psi}(\omega)=\sqrt{2 \pi} \omega^{2} e^{-\frac{\omega^{2}}{2}}
$$


Then,

$$
\begin{aligned}
\lim _{l \rightarrow \infty} \frac{1}{l} \int_{0^{+}}^{l}\left|\hat{\psi}_{a, b}(\lambda-\omega)\right|^{2} \frac{d a}{a} & =\lim _{l \rightarrow \infty} \frac{1}{l} \int_{0^{+}}^{l}|\hat{\psi}(a(\lambda-\omega))|^{2} d a \\
& =\lim _{l \rightarrow \infty} \frac{2 \pi}{l} \int_{0^{+}}^{l} a^{4}(\lambda-\omega)^{4} e^{-a^{2}(\lambda-\omega)^{2}} d a \\
& =\lim _{l \rightarrow \infty} \frac{3 \pi}{2} \frac{1}{l} \int_{0^{+}}^{l} e^{-a^{2}(\lambda-\omega)^{2}} d a \\
& =\frac{3 \pi}{2} \delta(\lambda-\omega) .
\end{aligned}
$$

\section{Local fractal dimension}

As suggested by Pentland (1984), we will consider a fractal random field $X$ with spectral density of the form

$$
f\left(\omega_{1}, \omega_{2}\right)=\frac{\sigma^{2}}{\left(\omega_{1}^{2}+\omega_{2}^{2}\right)^{2 \beta-\frac{1}{2}}}, \beta>\frac{1}{4}, \sigma, \omega_{1}, \omega_{2} \in \Re
$$

as $\omega_{1}^{2}+\omega_{2}^{2} \rightarrow 0$ to represent natural image data. As shown in Anh et al. (1996), (10) has the approximation

$$
f_{D}\left(\omega_{1}, \omega_{2}\right) \sim \frac{\sigma^{2}}{4 \pi^{2}}\left(\omega_{1}^{2}+\omega_{2}^{2}\right)^{\frac{1}{2}-2 \beta}, \omega_{1}, \omega_{2} \in[-\pi, \pi]
$$

as $\omega_{1}^{2}+\omega_{2}^{2} \rightarrow 0$. Noting that

$$
\left|4-\left(e^{i \omega_{1}}+e^{-i \omega_{1}}+e^{i \omega_{2}}+e^{-i \omega_{2}}\right)\right|^{2}=\left[4 \sin ^{2} \frac{\omega_{1}}{2}+4 \sin ^{2} \frac{\omega_{2}}{2}\right]^{2} \rightarrow\left(\omega_{1}^{2}+\omega_{2}^{2}\right)^{2}
$$

near the origin, the approximation (11) suggests the model

$$
\begin{gathered}
f_{D}\left(\omega_{1}, \omega_{2}\right)=\frac{\sigma^{2}}{4 \pi^{2}} \frac{1}{\left|4-\left(e^{i \omega_{1}}+e^{-i \omega_{1}}+e^{i \omega_{2}}+e^{-i \omega_{2}}\right)\right|^{2 d}} \\
d=\beta-\frac{1}{4}
\end{gathered}
$$

as a discrete approximation to (10) near the origin. As a result, Proposition 2 of Anh et al. (1996) implies that the dimension of the random field $X$ with spectral density (10) can be inferred as

$$
\operatorname{dim}(\operatorname{graph}(X))=3-2 d .
$$

The problem now is to estimate $d$ from the data, hence yielding the fractal dimension of $X(t)$ via (13). It is seen from (12) that this can be done via the regression

$$
\begin{aligned}
\ln f_{D}\left(\omega_{1 j}, \omega_{2 j}\right)= & a-d \ln \left|4-\left(e^{i \omega_{1 j}}+e^{-i \omega_{1 j}}+e^{i \omega_{2 j}}+e^{-i \omega_{2 j}}\right)\right|^{2} \\
& +u\left(\omega_{1 j}, \omega_{2 j}\right)
\end{aligned}
$$


where $a$ is the constant term, $\omega_{i j}=\frac{2 \pi j}{T}, 1 \leq j<T, T$ being the sample size (e.g. $T=5$ ), $i=1,2$, and $u$ is planar white noise. For an estimate of $f_{D}$, Maeda et al. (1996) used the 1-D version of the local periodogram (9) based on a $B$ spline wavelet, which is a good approximation to the 1-D Marr wavelet. It is noted that a fast algorithm for a 2-D discrete wavelet transform is not available (unless the separability condition is assumed, in which case this is performed as a composition of two 1-D wavelet transforms). Hence, in this paper, in addition to the 1-D version of the local periodogram (9), we also estimate $f_{D}$ by the usual periodogram, which is computed via the 2-D FFT. The result will then be compared with the 1-D Fourier-wavelet approach.

\section{Segmentation procedure}

Our segmentation procedure integrates the region information in the form of the FD map and the edge information in the form of the edge map on boundary pixels obtained from an original image by boundary Sobel operator. The edge information in the region growing algorithm plays an important role to decide whether the two adjacent regions should be merged or not. If there is no edge between the current region and the adjacent neighbouring region, the neighbouring region is merged into the current region. If there is an edge between them, the growth toward that direction is terminated. When the growth towards all possible directions is completed, one segmented region including the current region is formed. These operations are repeatedly performed for the complete image.

The steps of the algorithm are as follows:

1. The FD map and the edge map are computed from an original image. The FD map is calculated from a $3 \times 3$ local area for each pixel of the original image in the 1-D Fourier-wavelet method and from a $5 \times 5$ local area for the 2-D Fourier method. The edge map is obtained by the boundary Sobel operator and used after thresholding.

2. The original image is divided into initial kernel regions according to the region growing algorithm based on the grey levels of the original image. The neighbouring pixel is merged into the initial kernel region $R_{0}$ if

$$
\left|g_{\text {ave }}\left(R_{0}\right)-g(i, j)\right| \leq T_{G L}
$$

is satisfied, where $g_{a v e}\left(R_{0}\right)$ is the average grey level within the kernel region $R_{0}, g(i, j)$ is the grey value of the neighbouring pixel at $(i, j)$, and $T_{G L}$ is a predetermined threshold.

3. Starting from the initial kernel regions, the region growing algorithm is carried out based on the FD information and the edge information. A neighbouring pixel is merged into the intial kernel region $R_{0}$ if both of the following two conditions are satisfied:

- There is no edge between the initial kernel region $R_{0}$ and the neighbouring pixel at $(i, j)$ that is located in the direction of the growth. 
- $\left|d\left(R_{0}\right)-d(i, j)\right| \leq T_{F D}$, where $d\left(R_{0}\right)$ is the average FD value within the initial kernel region $R_{0}, d(i, j)$ is the FD of the neighbouring pixel at $(i, j)$, and $T_{F D}$ is a predetermined threshold.

The above region growing algorithm is executed for all neighbouring pixels around the initial kernel regions, and a newly merged region is created.

4. The region growing algorithm is carried out by repeatedly performing Step 3 until there is no remaining region that is to be merged in the whole image.

5. The smaller regions that remain after the above steps are removed as noise under the following conditions: Calculate the differences between the average FD values within the small region under consideration and the average FD values within all neighbouring regions around the small region. Select one region from several neighbouring regions that have the smallest value of the above differences. If the smallest difference is less than the threshold value $T_{F D}$, the small region is merged into the selected neighbouring region. In other words, the small regions are removed.

\section{Experimental results}

Figure 1 shows an original natural image that has $400 \times 400$ pixels and 256 grey levels. The local FD maps calculated from the original image using the 1-D Fourier-wavelet method and the 2-D Fourier method are shown in Figures 2 and 3 respectively. These figures indicate that the FD maps from both methods are still very detailed, but invariant to the lighting condition (e.g. shading); the wood texture of the two walls of the house is captured clearly although one wall is in the shade in the original image. This is an advantage of using the FD as a key feature in a segmentation scheme.

The segmented images by the region-edge integrating algorithm based on the FD maps of Figures 2 and 3 are shown in Figures 4 and 5 respectively. Both methods provide good segmentation and are quite comparable to each other. The circular signs on the two walls show clearer details in the 2-D Fourier method. This will be advantageous in applications such as road sign recognition, where the only relevant details come from the road sign which must be recognised in a natural scene. It seems that a segmentation with less detail but higher accuracy can be achieved if proper image processing is applied to the FD map of the 2-D method prior to executing the segmentation algorithm, since this FD map (Fig. 3) possesses sufficient information for this purpose. Furthermore, the 2-D FFT is relatively fast, while the Fourier-wavelet technique requires more processing time, since a discrete wavelet transform must by performed for each frequency used in the regression for estimating the local FD (see Eq. (12) of Maeda et al. (1996)).

\section{Conclusions}

This paper has put forward a method for the segmentation of natural images that integrates the FD information and the edge information into a region grow- 
ing algorithm. We have proposed two highly-accurate methods to estimate the local FD based on a 1-D Fourier-wavelet transform and a 2-D Fourier transform. We have also investigated the boundary edge in order to improve the control ability in the region-edge integrating algorithm, and to simplify the segmentation algorithm. A comparison has been made between the 1-D Fourier-wavelet method and the 2-D Fourier method. Experimental results indicate that both methods provide promising results in the segmentation of natural images.

\section{References}

1. Anh, V.V., Gras, F. and Tsui, H.T., Multifractal description of natural scenes, Fractals 4 (1996) 35-43.

2. Keller, J.M., Chen, S. and Crownover, R., Texture description and segmentation through fractal geometry, Computer Vision, Graphics and Image Processing 45 (1989) $150-166$.

3. Keller, J.M. and Seo, Y.B., Local fractal geometric features for image segmentation, Int. J. Imaging Systems and Technology 2 (1990) 267-284.

4. Koornwinder, T.H., Wavelets: An Elementary Treatment of Theory and Applications, World Scientific, Singapore, 1993.

5. Maeda, J., Anh, V.V., Ishizaka, T. and Suzuki, Y., Integration of local fractal dimension and boundary edge in segmenting natural images, Proc. IEEE Int. Conf. on Image Processing, P. Delogne (Ed.), Ceuterick, Leuven, 1996, Vol. 1, 845-848.

6. Pavlidis, T. and Liow, Y.T., Integrating region growing and edge detection, IEEE Trans. PAMI 12 (1990) 225-233.

7. Pentland, A.P., Fractal-based description of natural scenes, IEEE Trans. PAMI 6 (1984) $661-674$.

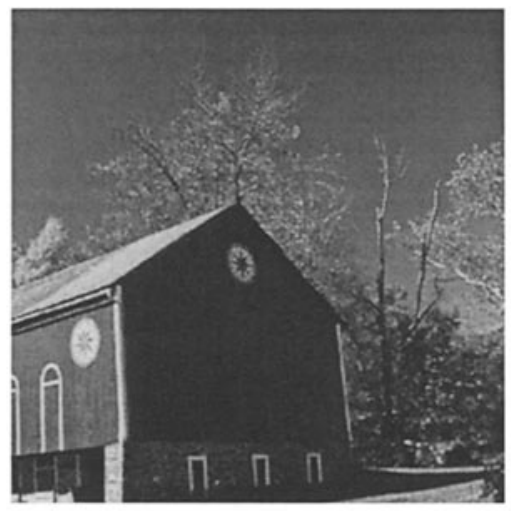

Fig. 1. The original image. 


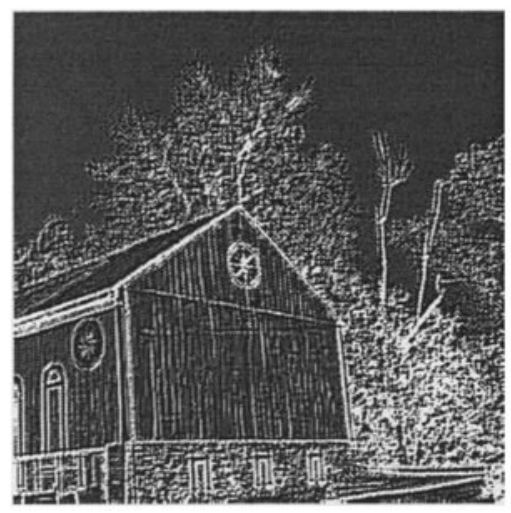

(a)

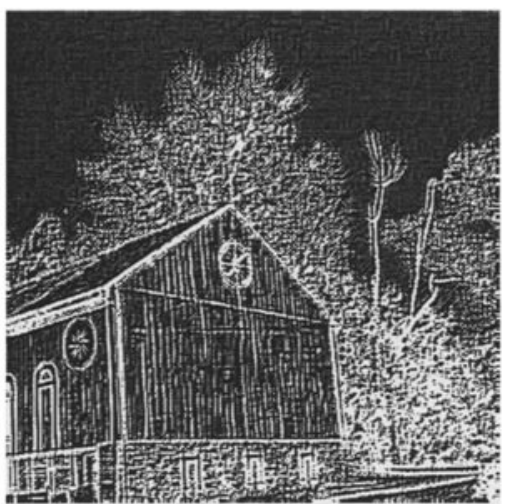

(b)

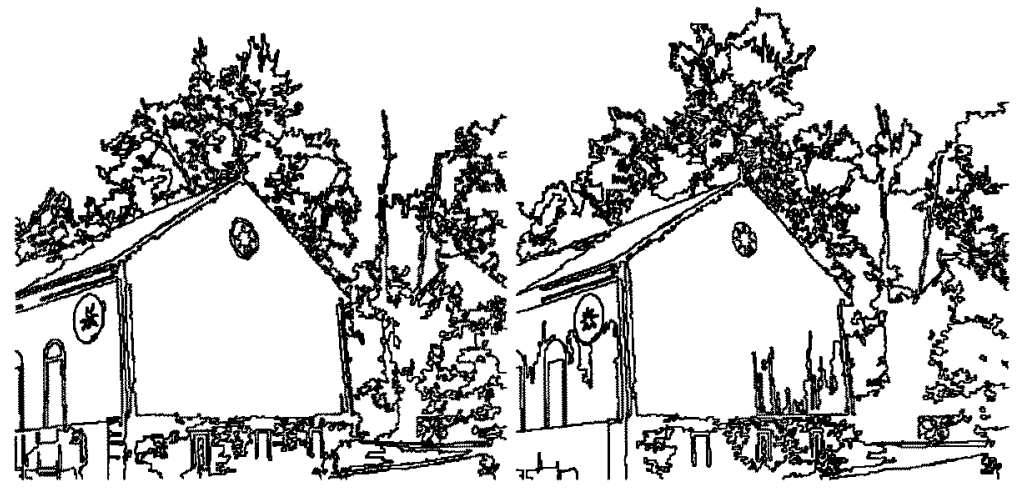

(c)

(d)

Fig. 2. (a)The FD map using the 1-D Fourier-wavelet method with $3 \times 3$ local blocks; (b)The FD map using the 2-D Fourier method with $5 \times 5$ local blocks; (c)The segmented image based on the FD map of (a); (d)The segmented image based on the FD map of (b). 